\title{
Addition of serum-containing medium to cerebrospinal fluid prevents cellular loss over time
}

\author{
Marieke T. de Graaf • Patricia D. M. van den Broek • Jaco Kraan • \\ Ronald L. Luitwieler $\cdot$ Martin J. van den Bent $\cdot$ Joke G. Boonstra • \\ Paul I. M. Schmitz · Jan W. Gratama - Peter A. E. Sillevis Smitt
}

Received: 6 December 2010/Revised: 19 February 2011/Accepted: 21 February 2011/Published online: 12 March 2011

(C) The Author(s) 2011. This article is published with open access at Springerlink.com

\begin{abstract}
Immediately after sampling, leukocyte counts in native cerebrospinal fluid (CSF) start to decrease rapidly. As the time lapse between CSF collection to analysis is not routinely registered, the clinical significance of decreasing cell counts in native CSF is not known. Earlier data suggest that addition of serum-containing medium to CSF directly after sampling prevents this rapid decrease in leukocyte counts and, thus, may improve the accuracy of CSF cell counting and cell characterization. Here, we prospectively
\end{abstract}

Electronic supplementary material The online version of this article (doi:10.1007/s00415-011-5970-8) contains supplementary material, which is available to authorized users.

M. T. de Graaf · M. J. van den Bent · P. A. E. Sillevis Smitt ( $\square)$ Department of Neurology, Erasmus University Medical Center, Room H-639, 's-Gravendijkwal 230, 3015 CE Rotterdam,

The Netherlands

e-mail: p.sillevissmitt@erasmusmc.nl

M. T. de Graaf · P. D. M. van den Broek · J. Kraan .

J. W. Gratama

Department of Medical Oncology,

Erasmus University Medical Center,

Rotterdam, The Netherlands

R. L. Luitwieler

Department of Anesthesiology,

Sint Franciscus Gasthuis, Rotterdam,

The Netherlands

J. G. Boonstra

Department of Clinical Chemistry,

Erasmus University Medical Center,

Rotterdam, The Netherlands

P. I. M. Schmitz

Department of Trials and Statistics,

Erasmus University Medical Center,

Rotterdam, The Netherlands examined the effect of storage time after lumbar puncture on counts of leukocytes and their major subsets in both native CSF and after immediate addition of serum-containing medium, measured by flow cytometry and microscopy. We collected CSF samples of 69 patients in tubes with and tubes without serum-containing medium and determined counts of leukocytes and subsets at 30 minutes, 1 hour, and 5 hours after sampling. Compared to cell counts at 30 minutes, no significant decrease in cell number was observed in CSF with serum-containing medium 1 and 5 hours after sampling, except for the granulocytes at 1 hour. In native CSF, approximately $50 \%$ of leukocytes and all their subsets were lost after 1 hour, both in flow cytometric and microscopic counting. In 6/7 (86\%) samples with mild pleocytosis $\left(5-15 \times 10^{6}\right.$ leukocytes/l), native CSF at 1 hour was incorrectly diagnosed as normocellular. In conclusion, addition of serum-containing medium to CSF directly after sampling prevents cell loss and allows longer preservation of CSF cells prior to analysis, both for microscopic and flow cytometric enumeration. We suggest that this protocol results in more accurate CSF cell counts and may prevent incorrect conclusions based on underestimated CSF cell counts.

Keywords Cerebrospinal fluid - Leukocytes . Flow cytometry $\cdot$ Lumbar puncture

\section{Introduction}

Cerebrospinal fluid (CSF) pleocytosis $\left(>5 \times 10^{6}\right.$ leukocytes/l) may indicate an infectious or inflammatory etiology of neurological symptoms [7]. Examples include viral, tuberculous and aseptic meningitis, neurosarcoidosis, neuro-Behçet, and paraneoplastic neurological syndromes. 
Earlier studies showed that leukocytes, derived from blood or CSF, disappear rapidly when spiked into CSF $[3,6,12]$. After 2 hours of storage in CSF, a leukocyte loss of $15-62 \%[3,6,12]$ was observed, while after storage for 24 hours only $39-60 \%$ of leukocytes remained detectable [3]. Furthermore, the delay from sampling to processing has a differential effect on the various cell types in CSF and affects the number of granulocytes most $[6,12]$.

However, the time from CSF collection to analysis is not routinely registered, and the clinical significance of decreasing cell counts in native CSF is not known. The rapid cellular loss in CSF suggests that CSF of patients with mild pleocytosis may be falsely diagnosed as normocellular depending on the time between lumbar puncture (LP) and analysis of the sample. Addition of serum-containing medium to CSF may prevent the rapid loss of leukocytes and their subsets [13] and, thereby, improve the accuracy of CSF cell counting and characterization.

In addition to the delay between sampling and analysis, CSF cell counts may also be affected by the counting method. Manual or microscopic analysis is the gold standard for determination of the (differential) white blood cell (WBC; leukocyte) and red blood cell (RBC; erythrocyte) counts in CSF. However, this technique is very time-consuming, costly, and prone to intertechnician variability and low precision $[1,2]$. Flow cytometry appears to improve accuracy and precision of CSF cell counting [5, 9]. Moreover, flow cytometry permits differentiation of leukocytes into lymphocytes and their subsets, monocytes and granulocytes by immunophenotyping, whereas microscopic analysis only differentiates between mononuclear cells (MNC; lymphocytes and monocytes) and polymorphonuclear cells (PMN; granulocytes).

In this study, we prospectively examined the effect of storage time after LP on counts of leukocytes and their major subsets in both native CSF and after addition of serum-containing medium, as measured by flow cytometry and microscopy. The medium was added directly after LP as it is known that cell decline starts immediately after CSF sampling [3, 6, 11-13]. We determined the percentage of CSF samples with mild pleocytosis that would be incorrectly diagnosed as normocellular when only native CSF was examined. This study was conducted on CSF samples of 69 patients.

\section{Methods}

\section{Patients}

A total of 61 patients with suspected neurological disease were included between January and August 2009 at the Department of Neurology, Daniel den Hoed Cancer Clinic,
Erasmus MC, Rotterdam. We included patients with suspected or proven leptomeningeal localization of a hematological or solid malignancy undergoing diagnostic or therapeutic LP in our in- or outpatient clinic. Patients were included only once. In addition, 8 patients without neurological disease undergoing spinal anesthesia for orthopedic, gynecological, or general surgery were included between May and August 2008 at the Department of Anesthesiology, Sint Franciscus Gasthuis, Rotterdam. The local ethical committees approved the study design and written informed consent was obtained from all patients.

\section{CSF collection}

Up to $5 \mathrm{ml}$ extra CSF was obtained from the 61 neurological patients during routine LP and aspirated before administration of the anesthetic drug in the 8 anesthesiological patients. Immediately after withdrawal, the CSF was split into different sterile tubes with or without serumcontaining medium (1:1 dilution) and stored at room temperature. The medium consisted of RPMI-1640 with $25 \mathrm{mM}$ HEPES, $1 \mathrm{mM}$ L-Glutamine, 2\% penicillin/streptomycin, 5\% heat-inactivated fetal bovine serum (FBS), and 2,500 IU heparin. This medium is routinely used in our laboratory for preservation of CSF cells before analysis $[6,10]$ and is stored in refrigerators in our in- and outpatient clinical wards where LPs are performed. All reagents were obtained from Cambrex Biosciences (Walkersville, MD), except for FBS (Greiner Bio-One, Frickenhausen, Germany) and heparin (Leo Pharma A/S, Ballerup, Denmark). In CSF of the 61 neurological patients, cell numbers were determined by flow cytometric and microscopic counting at 30 minutes, 1 hour, and 5 hours after sampling, while in CSF of the 8 patients who underwent spinal anesthesia, cell numbers were counted by flow cytometry only at 1 and 5 hours after sampling, due to smaller sample sizes in these patients.

Flow cytometric counting

Numbers of leukocytes, lymphocytes, monocytes, and granulocytes were determined by 6-color flow cytometry. CSF cells were concentrated by centrifugation ( 8 minutes, $450 \mathrm{~g}$ ) and resuspended in phosphate-buffered saline (PBS). Next, the sample was incubated for 15 minutes at room temperature (RT) in the dark with $10 \mu \mathrm{l}$ of each of the following monoclonal antibodies (mAb): $\mathrm{CD} 3$ conjugated with fluorescein isothiocyanate (FITC; clone SK7), CD56 conjugated with phycoerythrin (PE; clone C5.9), CD45 conjugated with peridinyl cholorophyllin (PerCP; clone 2D1), CD4 conjugated with PE-Cy7 (clone SK3), CD19 conjugated with allophycocyanin (APC; clone HIB19), and CD8 conjugated with APC-Cy7 (clone SK1). 
All mAb were obtained from BD Biosciences (San Jose, CA) with the exception of CD56-PE (Dako, Glostrup, Denmark) and CD19-APC (eBioscience, San Diego, CA). After incubation, cells were washed and subsequently resuspended in PBS. Immediately upon staining, list mode data were acquired on a 6-color FACSCanto flow cytometer (BD Biosciences, San Jose, CA). Analysis was performed using FCS Express software (De Novo Software, Los Angeles, CA). In the flow cytometric analysis of this study, we focused on leukocytes, lymphocytes, monocytes, and granulocytes as microscopic enumeration could only differentiate between leukocytes, mononuclear cells (MNC; lymphocytes and monocytes), and granulocytes. The immunophenotypical definitions of leukocytes and their major subsets are shown in Table 1.

\section{Microscopic counting}

Microscopic counting was performed before washing or concentration of the CSF. RBCs and WBCs were counted in a Fuchs-Rosenthal counting chamber at 400x magnification. First, RBCs were counted in unstained CSF. Next, CSF was incubated with concentrated Türk reagent (crystal violet dissolved in acetic acid; both obtained from Merck, Darmstadt, Germany) (10:1) for 5 minutes to lyse the RBCs and stain the WBCs. After incubation, WBCs were counted and visual differentiation between MNC and PMN was made. Subsequently, cell concentrations were calculated and corrected for dilution in medium.

\section{Statistics}

Because of the large variation in cell counts between samples, we used ratios to present the data clearly. Specifically, we calculated ratios for medium (cell count in native CSF divided by cell count in CSF with medium) in paired samples, for flow cytometry and for microscopy. These ratios were presented in box-and-whisker plots. To present the effect of storage time, the cell survival in native CSF and CSF with medium was determined ((i) cell count at 1 hour divided by cell count at 30 minutes, and (ii) cell count at 5 hours divided by cell count at 30 minutes) and

Table 1 Immunophenotypical definitions of leukocytes and their subsets

\begin{tabular}{ll}
\hline Subset & Immunophenotypical definition \\
\hline Leukocytes & $\mathrm{CD} 45^{+}$ \\
Lymphocytes & $\mathrm{CD} 45^{+}, \mathrm{SSC}^{\text {lo }}, \mathrm{FSC}^{\text {im }}$ \\
Monocytes & $\mathrm{CD} 45^{+}, \mathrm{SSC}^{\text {im }}, \mathrm{FSC}^{\text {hi }}, \mathrm{CD} 4{ }^{\text {dim }}$ \\
Granulocytes & $\mathrm{CD} 45^{+}, \mathrm{SSC}^{\text {hi }}$ \\
\hline
\end{tabular}

$S S C$ side scatter, FSC forward scatter, $h i$ high expression, im intermediate expression, dim dim expression, lo low expression graphically presented. Groups were compared with the Wilcoxon matched-pair test (SPSS version 15.0, SPSS Inc., Chicago, IL). Differences between groups with a $P$-value $<0.05$ were considered significant.

\section{Results}

Patients

Patient characteristics are presented in Table 2. Due to limited volumes of CSF in some patients, not all counting methods could be performed at all three time points within each sample. A total of $48 \mathrm{CSF}$ samples were investigated at 30 minutes with both flow cytometry and microscopy, while at 1 hour flow cytometry was performed on 69 samples and microscopy on 61 samples. At 5 hours after sampling, $27 \mathrm{CSF}$ samples were studied by flow cytometry and 19 by microscopy. Median and 25-75th percentiles of paired flow cytometric enumerations are presented in the Supplemental Table (published online).

\section{Effect of storage time on CSF cell counts}

Because it is not feasible to count cells directly after sampling, we considered the cell count at 30 minutes as a reasonable earliest measurement. Based on earlier data $[6,10,13]$, we postulated that addition of serum-containing

Table 2 Patient characteristics

\begin{tabular}{ll}
\hline Variable & \\
\hline $\mathrm{N}$ & 69 \\
Age: median (range) & $53(20-77)$ \\
Gender (M/F) & $42 / 27$ \\
Cerebrospinal fluid: median (range) & \\
Glucose (mmol/l) & $3.5(1.8-9.5)$ \\
Protein $(\mathrm{g} / \mathrm{l})^{*}$ & $0.34(0.05-2.47)$ \\
Lactate dehydrogenase $(\mathrm{U} / \mathrm{l})^{*}$ & $43(10-116)$ \\
RBC $\left(\times 10^{6} / \mathrm{l}\right)$ & $0(0-1,000)$ \\
WBC $\left(\times 10^{6} / 1\right)^{*}$ & $2(0-356)$ \\
Reason for lumbar puncture & 33 \\
Diagnostic & 28 \\
Therapeutic & 8 \\
Spinal anesthesia & \\
Malignancy & 43 \\
Hematological & 18 \\
Solid & 8 \\
No & \\
\hline$R B C$ red blood cells, $W B C$ white blood cells \\
Reference ranges: glucose $2.5-3.7$ mmol/l; protein $0.18-0.58 \mathrm{~g} / 1 ;$ \\
lactate dehydrogenase $0-69$ U/l; WBC $0-5 \times 10^{6} / 1$
\end{tabular}


medium immediately after CSF sampling, would prevent cell loss and provide the most accurate estimation of the number of cells in CSF at the time of sampling. We approximated this situation by performing the first enumerations at 30 minutes after sampling, a time lapse needed for transport of the CSF sample to the laboratory and setting up the counting procedure.

First, the effect of storage time after the addition of serum-containing medium to CSF was evaluated. At 1 and 5 hours after sampling, no significant decrease in the number of lymphocytes or monocytes counted by flow cytometry was observed in CSF with serum-containing medium as compared to 30 minutes after sampling (Fig. 1a). However, granulocytes did decrease significantly $(P<0.005)$, which may be due to their very low counts in our CSF samples (typically, CSF samples contained only $0.02-6.1 \times 10^{6}$ granulocytes/l vs. $0.01-43.3 \times 10^{6} \mathrm{lym}-$ phocytes/l). In native CSF (Fig. 1b), the leukocyte number declined significantly over time $(P<0.005)$, including the number of monocytes $(P<0.005)$ and granulocytes $\left(P<5 \times 10^{-6}\right)$. The number of lymphocytes was stable at both time points.

Addition of serum-containing medium immediately after CSF sampling

\section{Flow cytometry}

At all time points, flow cytometric counts of leukocytes and their subsets in native CSF were significantly lower than in CSF with serum-containing medium, except for the granulocyte count 30 minutes after sampling (Fig. 2). Furthermore, the ratio between cell counts in CSF without and with serum-containing medium decreased over time. After 5 hours, only $56 \%$ of the lymphocytes were left in native CSF (Fig. 2). The monocyte counts were affected even more: only $34 \%$ of cells was still detectable after 5 hours (Fig. 2). Most of the cell loss in native CSF occurred during the first hour after sampling, while the differences between 1 and 5 hours were not significant (data not shown).

At 1 hour after sampling, 15 of the 69 patients (22\%) had a pleocytosis $\left(5-344 \times 10^{6}\right.$ leukocytes/l) in the CSF sample with serum-containing medium. This pleocytosis was mild $\left(5-15 \times 10^{6}\right.$ leukocytes/l) in 7 patients. In the native samples, normal leukocyte counts were observed in 6 of the $15(40 \%)$ samples with pleocytosis and even in 6 of $7(86 \%)$ samples with a mildy increased leukocyte count.

\section{Microscopy}

Microscopic counts of WBC, MNC, and PMN (Fig. 3) were also significantly lower in native CSF compared to CSF with serum-containing medium at all time points, except for the PMN 30 minutes after sampling. When using microscopic counting, PMN showed the greatest loss in native CSF with a residual cell count of only $36 \%$ at 5 hours after sampling as compared to CSF stored in serum-containing medium.

\section{Discussion}

We prospectively investigated the effect of adding serumcontaining medium to CSF directly after sampling on cell counts determined by flow cytometry and microscopy in CSF samples from 69 patients. Our findings show that addition of serum-containing medium to CSF directly after sampling not only prevents immediate cell loss, but also allows longer preservation of CSF cells. The latter is
A CSF in serum-containing medium

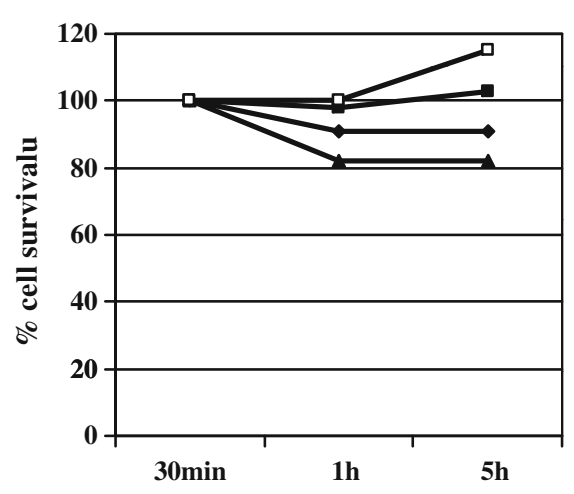

Fig. 1 Median percentage of cell survival of leukocytes and their subsets at 1 hour $(N=48)$ and 5 hours $(N=27)$ in $C S F$ stored in serum-containing medium (a) and native $\operatorname{CSF}(\mathbf{b})$ as determined by flow cytometry. The cell count at 30 minutes is set at $100 \%$. In CSF
B Native CSF

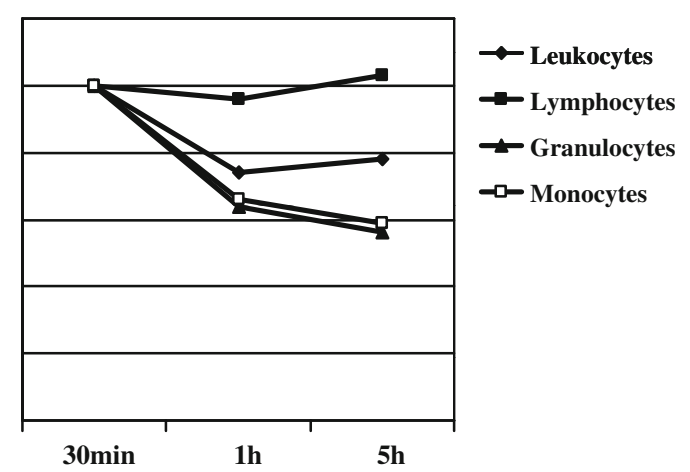

with medium the number of granulocyted declined significantly $(P<0.005)$ at 1 and 5 hours. In native CSF, the number of leukocytes $(P<0.005)$, monocytes $(P<0.005)$, and granulocytes $\left(P<5 \times 10^{-6}\right)$ declined significantly at 1 hour 

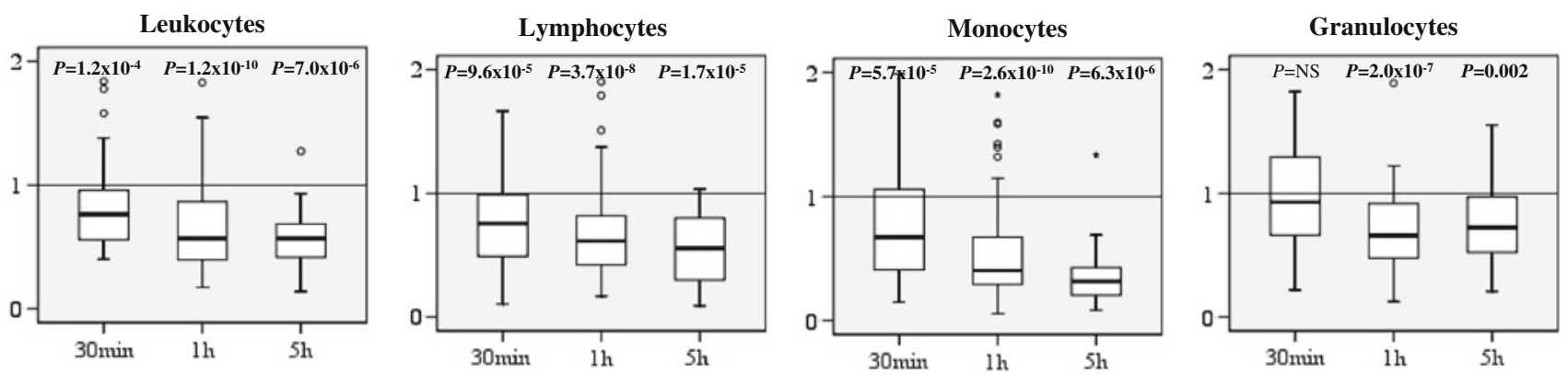

Fig. 2 Loss of cells in native CSF compared to CSF stored in serumcontaining medium as determined by flow cytometry. Ratios of cell count in native CSF divided by cell count in CSF with serumcontaining medium in paired samples for flow cytometric leukocyte, lymphocyte, monocyte, and granulocyte counts at 30 minutes $(N=48), 1$ hour $(N=69)$, and 5 hours $(N=27)$ after sampling. Box-and-whisker plots were used to present the data graphically,

showing median and interquartile ranges; the whiskers extended to the adjacent values, i.e., $1 \frac{1 / 2}{2} \times$ the interquartile range rolled back to where there is data. Observed points more extreme than the adjacent values were considered outliers and have been plotted individually. $P$ values (Wilcoxon matched-pair test) for the difference in cell counts between CSF with and without serum-containing medium are given

WBC

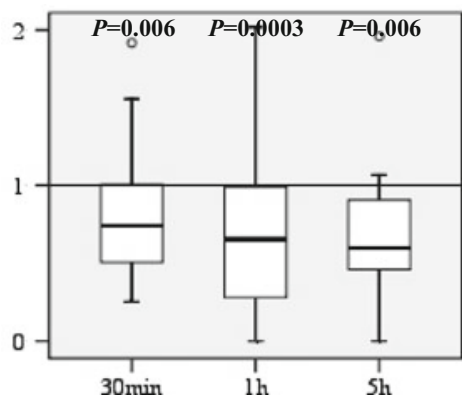

Fig. 3 Loss of cells in native CSF compared to CSF stored in serumcontaining medium as determined by microscopy. Ratios of cell count in native CSF divided by cell count in CSF with serum-containing medium in paired samples for microscopic white blood cell (WBC),

important for clinical practice. Especially after office hours, CSF samples may remain unattended at room temperature for considerable and unknown amounts of time: at a busy emergency room, during prolonged transportation towards the laboratory, and on the laboratory bench pending analysis.

In CSF with a mild pleocytosis, WBC counting in samples without addition of medium may result in a normal cell count and pathology, e.g., neuroinflammatory disease, may incorrectly be excluded. In our study, this situation was observed in $40 \%$ of the samples with a pleocytosis and even in $86 \%$ of the samples with a mildly increased WBC count. This result indicates that addition of serum-containing medium to CSF samples may prevent false-negative CSF interpretation and, thereby, impact clinical decision-making.

In addition to the total number of WBCs, we also studied the major WBC subsets. The selective loss of granulocytes, in native clinical CSF samples as well as in CSF with medium, has not been described earlier but is not
MNC

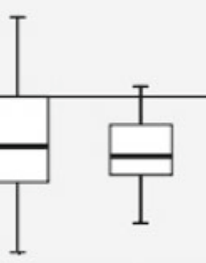

PMN

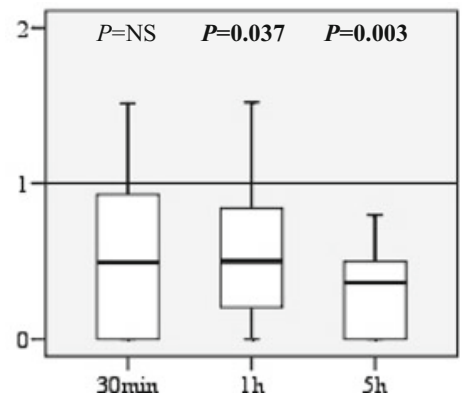

mononuclear cell $(M N C)$, and polymorphonuclear cell $(P M N)$ counts at 30 minutes $(N=48), 1$ hour $(N=61)$, and 5 hours $(N=19)$ after sampling are presented in box-and-whisker plots. See Fig. 2

unexpected as even under ideal circumstances granulocytes remain viable for only 7 hours [12]. Knowledge of this selective cell loss is important, because it may result in distortion of the relative size of cell populations [6] and, thereby, affect clinical decisions (e.g., predominance of granulocytes in CSF may differentiate early bacterial from aseptic meningitis).

Different media can be added to CSF to reduce the cell loss. Here, we show that addition of serum-containing medium preserves CSF cells until at least 5 hours after sampling. An earlier study showed that immediate addition of Earle's balanced salt solution with human serum albumin to CSF prevents total WBC loss until at least 24 hours after sampling [13]. In this study, major WBC subsets were not determined [13]. Other previous reports that investigated CSF cell preservation methods were more laboratory based than clinical. Spiking of CSF cells into 5\% fetal calf serum [6] or saline [12] showed no significant cell loss, while spiking into acellular CSF did $[6,12]$. In addition, refrigeration of the CSF sample [3] and a minimum of 
centrifugation steps [6] also reduce cell loss. The function of serum-containing media in preserving cells is probably a buffering one. Both increase in $\mathrm{pH}$ after removal of CSF from the body [4] and low osmolarity [8, 12] have been postulated to cause cell death. Also, it remains to be investigated which medium is the most effective in preventing CSF cell loss in clinical settings from practical and economic perspectives.

Based on the results of this study, we recommend adding serum-containing medium to CSF directly after collection, not only when CSF flow cytometry has to be performed, but also when cells are routinely counted by microscopy. Addition of serum-containing medium may prevent false-negative reports of normal cell counts in CSF samples with a mild pleocytosis. Although the conditions and the maximal storage time to preserve CSF samples for optimal cellular analyses still have to be optimized, we may safely conclude that the use of native CSF samples for such analyses should be discouraged.

Acknowledgments We would like to thank the neurologists and anesthesiologists for inclusion of the patients and the technicians of the Department of Clinical Chemistry in the Daniel den Hoed Cancer Clinic for their technical assistance.

\section{Conflict of interest None}

Open Access This article is distributed under the terms of the Creative Commons Attribution Noncommercial License which permits any noncommercial use, distribution, and reproduction in any medium, provided the original author(s) and source are credited.

\section{References}

1. Aulesa C, Mainar I, Prieto M, Cobos N, Galimany R (2003) Use of the Advia 120 hematology analyzer in the differential cytologic analysis of biological fluids (cerebrospinal, peritoneal, pleural, pericardial, synovial, and others). Lab Hematol 9:214-224
2. Aune MW, Sandberg S (2000) Automated counting of white and red blood cells in the cerebrospinal fluid. Clin Lab Haematol 22:203-210

3. Chow G, Schmidley JW (1984) Lysis of erythrocytes and leukocytes in traumatic lumbar punctures. Arch Neurol 41:1084-1085

4. Cunniffe JG, Whitby-Strevens S, Wilcox MH (1996) Effect of pH changes in cerebrospinal fluid specimens on bacterial survival and antigen test results. J Clin Pathol 49:249-253

5. de Graaf MT, Smitt PA, Luitwieler RL, van Velzen C, van den Broek PD, Kraan J, Gratama JW (2010) Central memory CD4(+) $\mathrm{T}$ cells dominate the normal cerebrospinal fluid. Cytometry $\mathrm{B}$ Clin Cytom

6. Dux R, Kindler-Rohrborn A, Annas M, Faustmann P, Lennartz K, Zimmermann CW (1994) A standardized protocol for flow cytometric analysis of cells isolated from cerebrospinal fluid. J Neurol Sci 121:74-78

7. Fishman RA (ed) (1992) Cerebrospinal fluid in diseases of the nervous system. Saunders Philadelphia, Pennsylvania

8. Fry MM, Vernau W, Kass PH, Vernau KM (2006) Effects of time, initial composition, and stabilizing agents on the results of canine cerebrospinal fluid analysis. Vet Clin Pathol 35:72-77

9. Kleine TO, Nebe CT, Lower C, Lehmitz R, Geilenkeuser WJ, Kruse R, Dorn-Beineke A (2010) Evaluation of cell counting and leukocyte differentiation in cerebrospinal fluid controls using hematology analyzers by the German Society for Clinical Chemistry and Laboratory Medicine. Clin Chem Lab Med 48:839-848

10. Kraan J, Gratama JW, Haioun C, Orfao A, Plonquet A, Porwit A, Quijano S, Stetler-Stevenson M, Subira D, Wilson W (2008) Flow cytometric immunophenotyping of cerebrospinal fluid. Curr Protoc Cytom ;chapter 6:unit 625

11. Quijano S, Lopez A, Manuel Sancho J, Panizo C, Deben G, Castilla C, Antonio Garcia-Vela J, Salar A, Alonso-Vence N, Gonzalez-Barca E, Penalver FJ, Plaza-Villa J, Morado M, GarciaMarco J, Arias J, Briones J, Ferrer S, Capote J, Nicolas C, Orfao A (2009) Identification of leptomeningeal disease in aggressive B-cell non-Hodgkin's lymphoma: improved sensitivity of flow cytometry. J Clin Oncol 27:1462-1469

12. Steele RW, Marmer DJ, O'Brien MD, Tyson ST, Steele CR (1986) Leukocyte survival in cerebrospinal fluid. J Clin Microbiol 23:965-966

13. Veerman AJ, Huismans L, van Zantwijk I (1985) Storage of cerebrospinal fluid samples at room temperature. Acta Cytol 29:188-189 